\title{
Efficiency and Effectiveness of State Transport Undertakings in India: A DEA Approach
}

\author{
Sanjay K. Singh, Amit P. Jha \\ Indian Institute of Management, Lucknow, India \\ Email: sanjay@iiml.ac.in,fpm16014@iiml.ac.in
}

How to cite this paper: Singh, S.K. and Jha, A.P. (2017) Efficiency and Effectiveness of State Transport Undertakings in India: A DEA Approach. Theoretical Economics Letters, 7, 1646-1659. https://doi.org/10.4236/tel.2017.76111

Received: August 21, 2017

Accepted: September 18, 2017

Published: September 21, 2017

Copyright @ 2017 by authors and Scientific Research Publishing Inc. This work is licensed under the Creative Commons Attribution International License (CC BY 4.0).

http://creativecommons.org/licenses/by/4.0/ (c) (i) Open Access

\begin{abstract}
The Indian bus transport industry is dominated by the publicly owned State Transport Undertakings (STUs). Most of the STUs have, over the years, accumulated financial losses. However, since STUs offer their services with a social aim, financial losses faced by them may not be bad per se. For publicly owned organizations, efficiency and effectiveness are more important than mere profitability. This paper attempts to measure the efficiency and effectiveness of fifteen major STUs in India for the period 2003-04 to 2013-14 using Data Envelopment Analysis (DEA). The paper also examines STUs' scale elasticity and its relationship with firm size. It is found that the STUs operating in the state of West Bengal are not only the least efficient but also the least effective whereas Andhra Pradesh state road transport corporation, which is the largest bus transport operator in the world, is the most efficient and effective operator. In general, there is a strong positive correlation between STUs' efficiency and their effectiveness. On the other hand, there is a negative relationship between size of the STUs and returns to scale; large size firms are showing decreasing returns to scale whereas small size ones are operating on increasing returns to scale. Therefore, a size correction through mergers, demergers or altering scale of operation, as the case may be, will be economically prudent.
\end{abstract}

\section{Keywords}

DEA, Efficiency, Effectiveness, Scale Elasticity, Indian STUs

\section{Introduction}

Road transport in India has gained importance over other modes of transportation during the last few decades. If we look at the history of development of modern modes of transportation in India, we notice a departure in the trend 
between the first half of the previous century and the latter. In pre-independence India, railway was the dominant mode of transport. Market share of railways started to decline since 1950-51 and the number of buses in India went up from a meagre 56,800 in 1960-61 to more than half a million by the turn of the century. The number of buses in India was about 1,887,000 during 2013-14, representing an average compounded annual growth rate of 6.8 percent since 1960-61.

In India, publicly owned bus transport companies, known as State Transport Undertakings (STUs), have an important role in passenger bus transport since private sector is highly fragmented. Presently, STUs in India are operating with 137,000 buses and employing close to 700,000 people. During the year 2013-14, latest year for which data are available, the total bus-kilometres operated by them were around 15 billion, the number of passengers carried was over 23 billion, and the volume of operations had crossed the mark of 500 billion passenger-kilometres. From the very beginning, STUs in India faced huge financial losses from their operation. STUs' total revenue during the year 2013-14 was just Rs. 431.19 billion in comparison to total cost of Rs. 502.31 billion. Due to this, they faced a net loss of more than Rs. 71 billion during the year 2013-14. On an average, every bus-km operated by these undertakings resulted in a loss of around Rs. 5 during the same year.

However, since STUs in India offer their services with a social aim, financial losses faced by them may not be bad per se. For publicly owned public transport organizations, efficiency and effectiveness are more important than mere profitability. Efficiency and effectiveness evaluation in public transportation is therefore an issue of foremost importance. There are several approaches to measure transport operators' efficiency and effectiveness. Parametric and nonparametric frontiers are the two main approaches for this (for comparison, see, [1] [2] [3] [4] [5]). It is well known that parametric techniques (such as, Stochastic Frontier Analysis or SFA) require a set of distributional assumptions which may or may not hold for the set of firms in question. SFA is based on the work of Aigner et al. and further enriched by other researchers such as Battese and Coelli [6] [7] [8]. On the other hand, non-parametric techniques such as index number approach and Data Envelopment Analysis (DEA) require no such assumptions regarding variable distribution. The DEA technique is based on the seminal work by Farrell [9]. DEA, in its present form, was developed almost four decades ago by Charnes et al. and since then is refined by successive researchers such as Banker et al., Lovell and Rouse and various others [10] [11] [12].

Benchmarking tools and productivity measuring methodologies are used by various researchers in transportation sector. Kumbhakar and Bhattacharya (1996) considered an econometric approach with a translog cost function for production technology to measure total factor productivity growth and technical change for thirty one publicly owned passenger bus companies in India during 1983-87 [13]. Jørgensen et al. (1997) estimated a stochastic cost frontier model for the Norwegian bus industry to estimate the efficiency. Interestingly, they 
found no significant differences in the efficiency between privately and publicly owned operators, though it is hard to generalize the findings to developing economies [14]. Viton (1997) applied DEA on a relatively large sample of both privately and publicly operated bus systems from the United States to study their efficiency. Two output and multiple input measures such as, average speed, average fleet age, fleet size, fuel consumed, staff employed in various divisions, etc., were used and efficiencies were estimated, though, only returns to scale characteristics and not quantitative estimates of the same were reported [15].

Using a frontier approach for cost inefficiencies in Indian state road transport undertakings, Jha and Singh (2001) made the analyses and concluded that smaller STUs are, in general, more efficient [16]. Singh and Venkatesh (2003) compared efficiency across STUs using a production frontier approach [17]. As the dynamics of the industry is rapidly changing a renewed in-depth analysis of Indian STUs is called for. Karlaftis (2003) concluded that the results of various analysis indicated that efficiency and returns to scale findings differ substantially depending on the evaluation methodology used [18]. This necessitates further analysis of Indian STUs using the most general DEA models by incorporating newly developed theoretical frameworks to applied research.

Boame (2004) has studied technical efficiencies of urban transit systems of Canada by using DEA with bootstrapping and the average technical efficiency of transit systems was found to be 78 per cent. Transit systems mostly were found to experience increasing returns to scale [19].

Matthew G. Karlaftis (2004) used DEA approach for evaluating the efficiency and the effectiveness of urban transit systems in the US context and has further used goal programming technique by utilising Charnes et al. (1996) methodology to estimate return to scale measures for groups of transit systems through multiplicative DEA. The method develops an empirical efficient production function via a Robustly Efficient Parametric Frontier in a two-stage approach [20] [21]. Odeck and Alkadi (2004) applied DEA to Norwegian rural and urban bus operators and also used nonparametric testing for efficiency and scale differences with respect to ownership, region of operation and scope of operation [22].

Sampio et al. (2008) have analysed Brazilian and some European transport systems using CRS assumption of DEA with three inputs and one output. A causal link between efficiency and system of tariffs was also established [23]. Saxena and Saxena (2010) used DEA to measure efficiencies of some of the Indian STUs. Scale efficiencies were calculated but no attempts were made to estimate scale elasticities [24].

Agarwal et al. (2010) estimated the technical efficiency of public transport sector in India for thirty five different STUs for the year 2004-2005 by employing CCR input-oriented DEA model. Fleet size, number of staff, fuel consumption and a measure for accidents were inputs and bus utilization, passenger $\mathrm{km}$ and load factor were the outputs. On the basis of the status of technical efficiency (TE), it was concluded that the performance of the STUs were good but not op- 
timal. The mean overall TE was found to be 83.26 per cent [25]. Jordá et al. (2012) studied, by using slack-based measures model, the technical efficiency of the Spanish urban bus companies for 2004-09 [26].

Relative performance of twenty six Indian public urban transport organizations with 19 criteria-grouped in 3 heads; operations, finance, and accidentbased-was carried out by Vaidya (2014). The author computed efficiency using the CCR DEA approach. Analytical Hierarchy Process was used before applying DEA to assign weights to each criteria group and finally, a Transportation Efficiency Number (TEN) was developed to quantify the overall performance [27]. Hanumappa et al. (2015) studied the premium bus services operated under Bangalore Metropolitan Transport Corporation using input oriented CCR model of DEA. Analysis indicated that most depots were efficient, but some routes have significant opportunities for further improvement [28]. Venkatesh and Kushwaha (2017) is a recent attempt to measure technical efficiency of passenger bus companies in India using non-radial DEA [29].

Only a few literature is available where STUs in India are evaluated using DEA and very few have used full potential of DEA. This paper attempts to estimate efficiency and effectiveness along with combined performance of a fairly representative sample of Indian STUs by using DEA on panel data. The paper further attempts to fill the existing literature gap by estimating scale elasticity measures for the STUs which are less explored in the literature. Further, paper attempts to establish a connection between STUs' size and returns to scale. This may help managers and policymakers to determine optimal size of the STUs.

The remainder of this paper is organized in the following manner: Section 2 deals with the theoretical framework used in the study. Section 3 describes the data and the sample STUs. The results are discussed in Section 4. Section 5 presents the conclusion of the study.

\section{Theoretical Framework}

DEA is a well-established analytical tool to make comparisons among Decision Making Units (DMUs). The methodology with its extensions has rich applicability in applied research. We have adopted Variable Returns to Scale (VRS) DEA model for this study. This general model was proposed by Banker et al. [11]. The model is though well-known, yet sparingly used by transportation researchers in Indian context. Most authors have preferred original model as proposed by Charnes et al. [10]. Original model worked under the assumption of Constant Returns to Scale (CRS). One of the objectives of our study is to estimate the Returns to Scale (RTS) experienced by the STUs. VRS model can be extended to estimate the RTS.

A Data Envelopment Analysis can be conducted either from the output orientation or from the input orientation. We have used output orientations in our analysis. We assume that we have $j=\{1, \cdots, n\}$ DMUs (STUs in our case). We further assume that the DMUs take $i=\{1, \cdots, m\}$ inputs and produce 
$r=\{1, \cdots, s\}$ outputs, say $x_{i j}$ be the $\mathrm{i}^{\text {th }}$ input by the $\mathrm{j}^{\text {th }} \mathrm{DMU}$ and $y_{r j}$ be the $\mathrm{r}^{\text {th }}$ output from the $\mathrm{j}^{\text {th }}$ DMU. All the DMUs are evaluated for each of the $t$ periods, where, $t=\{1, \cdots, T\}$. The efficiency for the DMUs can be obtained from the following linear program which is based on Banker et al. [11].

$$
\begin{gathered}
\text { minimize } \sum_{i=1}^{m} v_{i} x_{i 0}+\xi \\
\text { subject to } \sum_{i=1}^{m} v_{i} x_{i j}-\sum_{r=1}^{s} u_{r} y_{r j}+\xi \geq 0, j=1, \cdots, n \\
\sum_{r=1}^{s} u_{r} y_{r 0}=1
\end{gathered}
$$

$u_{r}, v_{i} \geq \phi$ and $\xi$ is unrestricted.

$\phi$ is a small non-Archimedian positive number.

In CRS model, the variable $\xi$ is dropped from the formulation. The performance indicator in output orientation is the reciprocal of the objective function value. The relative performances thus measured are technical efficiencies.

For the dataset considered in our analysis, we have $n=15$ DMUs, $\mathrm{m}=\{1,2,3\}$ inputs and $s=\{1,2\}$ outputs. We have considered two outputs: passenger-km and bus-km and three inputs: number of staff employed, total fuel consumed and number of buses held. Output oriented DEAs are applied once for combined output, i.e., both passenger-km and bus-km as outputs and once each for one output scenario, i.e., once each for passenger-km and bus-km. We have considered $T=11$ time periods. All the linear programming problems are solved for each of the $T=11$ time periods. We have coded the linear programming problems in one of the standard statistical software, R. In sync with Karlaftis (2004), we have also obtained efficiency-effectiveness matrix [20]. The output measure passenger-km captures effectiveness and the output measure bus-km corresponds to efficiency.

In the next stage we compute returns to scale measure for each firm and for each time period. To formalize the discussion on scale elasticity estimation, assume a firm employing a vector of inputs $X$ to produce a vector of outputs $Y$. Let, all inputs are subjected to proportional expansion of $\alpha$ and the corresponding maximum proportional expansion in all outputs be $\beta$, such that,

$$
\phi(\alpha X, \beta Y)=0
$$

By definition, a measure of scale elasticity is,

$$
\frac{\partial \beta}{\partial \alpha}=\epsilon(X, Y)
$$

Sahoo and Tone (2015) have utilized Panzar and Willig (1977) to obtain a quantitative estimate of scale elasticity based on DEA approach [30] [31]. The measure of scale elasticity for the firm, $k$ is given by,

$$
\epsilon_{o}\left(X_{k}, Y_{k}\right)=1-\frac{\xi}{D_{o}(x, y)}
$$


In the above equations $\epsilon$ denotes the elasticity measure and $D$ denotes the values of objective function in the corresponding linear programing formulation of the VRS model. The subscript o is for output orientation. Values of $\xi$ are obtained from the solutions of the linear program formulation of the DEA. We have calculated elasticities using above formula for each firm, in each time period and for the two output scenario. In this way, we obtained not only returns to scale characteristics but also a scale elasticity measure. A value of $\epsilon$ equal to (or very close to) 1 corresponds to constant returns to scale scenario, a value less than 1 corresponds to decreasing returns to scale scenario and a value greater than 1 corresponds to increasing returns to scale scenario. This is a quantitative estimate of returns to scale. We emphasize that for scale elasticity estimation we have used output oriented DEA because the formula for input oriented DEA may fail to give finite elasticity measure when the value of objective function of the DEA linear program and the scale characteristic variable $\xi$ are equal in magnitude but $\xi$ is negative in sign [30]. Next we partitioned our dataset, with two outputs, in three categories (Large, Medium and Small sized respectively), using k-means clustering for each year, in order to establish relationship between firm size and RTS estimate.

\section{The Data and the Sample STUs}

Annual data for a sample of fifteen STUs from 2003-04 to 2013-14 are used for this study. The primary source of data is Performance Statistics of STUs, 2003-04 to 2013-14 published for the Association of State Road Transport Undertakings (ASRTU), New Delhi by the Central Institute of Road Transport, Pune, India. Sample is based on availability of consistent data. Sample STUs include Andhra Pradesh State Road Transport Corporation (APSRTC), Maharashtra State Road Transport Corporation (MSRTC), Karnataka State Road Transport Corporation (KnSRTC), North West Karnataka Road Transport Corporation (NWKnRTC), Gujarat State Road Transport Corporation (GSRTC), Uttar Pradesh State Road Transport Corporation (UPSRTC), Rajasthan State Road Transport Corporation (RSRTC), State Transport Haryana (STHAR), South Bengal State Transport Corporation (SBSTC), Kadamba Transport Corporation Limited (KDTC), Orissa State Road Transport Corporation (OSRTC), Kerala State Road Transport Corporation (KSRTC), North Eastern Karnataka Road Transport Corporation (NEKnRTC), North Bengal State Transport Corporation (NBSTC) and Bihar State Road Transport Corporation (BSRTC). The descriptive statistics of the sample STUs for the period 2013-14 is presented in Table 1.

Sample STUs are publicly owned, operate throughout their respective jurisdiction (often throughout the state), mainly provide inter-city and mofussil (rural) bus transport services, and do business in the field of passenger transportation only, but differ in size and the level of output produced. The size of the sample STUs, as measured by bus-kilometres (BKm) in 2013-14, ranges from 7 million BKm for BSRTC to 2623 million BKm for APSRTC. Fleet strength of 
Table 1. Descriptive statistics of the sample STUs during 2013-14.

\begin{tabular}{cccccc}
\hline STUs & $\begin{array}{c}\text { Pass.-km } \\
\text { (million) }\end{array}$ & $\begin{array}{c}\text { Bus-km } \\
\text { (million) }\end{array}$ & $\begin{array}{c}\text { No. of } \\
\text { employees }\end{array}$ & $\begin{array}{c}\text { HSD } \\
\text { consumed (Kl) }\end{array}$ & $\begin{array}{c}\text { No. of buses } \\
\text { held }\end{array}$ \\
\hline APSRTC & 88,246 & 2623 & 122,176 & 432,800 & 22,145 \\
MSRTC & 52,576 & 2047 & 107,709 & 433,745 & 18,055 \\
KnSRTC & 35,463 & 988 & 35,379 & 216,966 & 8257 \\
NWKnRTC & 20,731 & 550 & 22,508 & 110,735 & 4615 \\
GSRTC & 33,607 & 1033 & 40,122 & 181,031 & 7852 \\
UPSRTC & 37,721 & 1175 & 26,723 & 182,101 & 9600 \\
RSRTC & 22,215 & 600 & 21,384 & 117,051 & 4674 \\
STHAR & 15,138 & 421 & 18,634 & 123,866 & 3816 \\
SBSTC & 1673 & 35 & 2030 & 9081 & 424 \\
KDTC & 866 & 29 & 2013 & 6719 & 542 \\
OSRTC & 958 & 29 & 697 & 4005 & 422 \\
KSRTC & 24,765 & 556 & 42,127 & 138,779 & 7831 \\
NEKnRTC & 15,312 & 458 & 19,128 & 93,571 & 4247 \\
NBSTC & 1456 & 36 & 3111 & 9456 & 688 \\
BSRTC & 268 & 7 & 920 & 1683 & 414 \\
\hline
\end{tabular}

STUs varies drastically, from 414 buses for BSRTC to 22,145 buses for APSRTC. Number of workers employed by STUs also varies from less than 1000 for BSRTC and OSRTC to more than 100,000 for APSRTC and MSRTC. In almost all respect, BSRTC is the smallest STU whereas APSRTC is the largest one. In fact, APSRTC is the world's largest bus transport operator.

The sample is fairly good representative of the publicly owned bus transport industry; sample STUs constitute two third of the publicly owned bus transport industry in India. In 2013-14, they operated with 93,582 buses which is more than two third of the industry fleet size. During the same year, sample STUs consumed 2877 million litres of HSD which is more than $70 \%$ of the industry consumption. The total staff employed by sample STUs was 464,661 in 2013-14, which is again nearly a two third of the total staff employed by all the STUs. Our sample thus covers almost two third of the entire state owned public transport sector. Furthermore, our sample is fairly good representative of the entire state owned public transport sector in the sense that the firm size varies from small STUs such as BSRTC and OSRTC to large STUs such as APSRTC and MSRTC. The sample also includes medium size STUs such as GSRTC, UPSRTC and RSRTC.

We have considered a two output and three input model. Passenger-km and bus-km are our two of the outputs and labour/staff employed, fuel consumed and number of vehicles used are our input measures. All the data points are measured on a per year basis. Outputs are measured in million passenger-km 
and million bus-km, respectively. Inputs are measured as the number of staff employed; fuel consumption, measured as HSD consumed in kilo-litres; and number of vehicles, measured as the number of buses held by the respective STUs. Some of the STUs such as those operating in north eastern states and Tamil Nadu-where STUs are fragmented-were not taken in the sample because of at least two reasons. Firstly, all the required data fields for the entire time series under consideration were not available, perhaps because of non-reporting by the concerned STU. Secondly, smaller STUs may lead to bias in the analysis.

Charnes et al. (1996) have suggested that the number of DMUs in a DEA should be at least thrice the number of variables considered. In the DEA literature, number of inputs, number of outputs and the total number of DMUs considered are represented by $\mathrm{m}, \mathrm{s}$ and $\mathrm{n}$, respectively. In our case, $m=3, s=2$, and $n=15$. So, the criteria $n \geq 3(m+s)$ is satisfied [21].

\section{The Results}

Performance of the STUs can be viewed either based on passenger-km or bus-km (Matthew G Karlaftis, 2004) along with an overall performance [20]. Whereas, higher bus-km for a given input set may be called more efficient, a still better view is the passenger-km based. It is the passenger-km which is of prime importance in public transport and hence a higher value for a given input set corresponds to higher effectiveness. To evaluate these, we have employed DEA with one output-either passenger-km or bus-km-along with an overall performance based on combined output model. Relative performances along with the temporal changes in the cross sectional performances have been examined. Analysis has been performed based on the annual data for the entire time period considered. The efficiency and the effectiveness scores are reported for selected years in Table 2. Mean efficiency and mean effectiveness scores are calculated based on arithmetic average of the annual scores and STUs are ranked based on the same.

Table 2 results show that substantial inefficiencies exist in few STUs; average inefficiency in NBSTC is $33.48 \%$ followed by KSRTC (28.30\%), SBSTC (21.06\%), BSRTC (11.89\%), and MSRTC (10.26\%). However, three STUs of APSRTC, RSRTC, and OSRTC, experienced efficiency scores of $100 \%$ during the sample period. Average inefficiency in remaining seven STUs is less than $10 \%$, varying from $1.04 \%$ for USRTC to $8.83 \%$ for KDTC. As far as effectiveness is concerned, two STUs, APSRTC and OSRTC, are the most effective ones with $100 \%$ score during the sample period. As is the case with efficiency, average effectiveness score also varied substantially; NBSTC is not only the least efficient but also the least effective STU. Average ineffectiveness score of NBSTC is $33.40 \%$ followed by MSRTC (27.18\%), SBSTC (25.80\%), KDTC (15.66\%), NWKnRTC (14.3\%), BSRTC (12.82\%), KSRTC (12.71\%), GSRTC (11.22\%), NEKnRTC (11.09\%), and STHAR (10.49\%). It is interesting to note that the average ineffectiveness score exceeds $10 \%$ for ten STUs whereas average inefficiency score exceeds $10 \%$ for 
Table 2. Efficiency and effectiveness scores of sample STUs based on a single output.

\begin{tabular}{|c|c|c|c|c|c|c|c|c|c|c|}
\hline \multirow{2}{*}{ STU } & \multicolumn{3}{|c|}{ Efficiency Scores (\%) } & \multirow[b]{2}{*}{ Mean (2003-14) } & \multirow{2}{*}{$\begin{array}{c}\text { Efficiency } \\
\text { Ranking }\end{array}$} & \multicolumn{3}{|c|}{ Effectiveness Scores (\%) } & \multirow[b]{2}{*}{ Mean (2003-14) } & \multirow{2}{*}{$\begin{array}{c}\text { Effectiveness } \\
\text { Ranking }\end{array}$} \\
\hline & 2003-04 & 2008-09 & 2013-14 & & & 2003-04 & 2008-09 & $2013-14$ & & \\
\hline APSRTC & 100.00 & 100.00 & 100.00 & 100.00 & 1 & 100.00 & 100.00 & 100.00 & 100.00 & 1 \\
\hline MSRTC & 91.49 & 87.85 & 94.41 & 89.74 & 11 & 79.73 & 68.96 & 72.23 & 72.82 & 14 \\
\hline KnSRTC & 100.00 & 93.12 & 93.30 & 95.48 & 6 & 81.57 & 97.06 & 100.00 & 97.47 & 3 \\
\hline NWKnRTC & 100.00 & 87.53 & 92.42 & 91.95 & 8 & 77.51 & 72.38 & 97.49 & 85.70 & 11 \\
\hline GSRTC & 92.89 & 100.00 & 100.00 & 96.47 & 5 & 70.23 & 90.43 & 98.19 & 88.78 & 8 \\
\hline UPSRTC & 98.91 & 100.00 & 100.00 & 98.96 & 4 & 71.16 & 91.55 & 100.00 & 92.07 & 5 \\
\hline RSRTC & 100.00 & 100.00 & 100.00 & 100.00 & 1 & 67.36 & 100.00 & 100.00 & 96.73 & 4 \\
\hline STHAR & 93.93 & 97.12 & 86.20 & 91.85 & 9 & 65.17 & 100.00 & 83.78 & 89.51 & 6 \\
\hline SBSTC & 81.36 & 78.72 & 100.00 & 78.94 & 13 & 55.05 & 70.93 & 100.00 & 74.20 & 13 \\
\hline KDTC & 100.00 & 98.08 & 63.58 & 91.17 & 10 & 94.14 & 85.07 & 58.04 & 84.34 & 12 \\
\hline OSRTC & 100.00 & 100.00 & 100.00 & 100.00 & 1 & 100.00 & 100.00 & 100.00 & 100.00 & 1 \\
\hline KSRTC & 77.60 & 71.57 & 61.96 & 71.70 & 14 & 100.00 & 76.47 & 86.05 & 87.29 & 9 \\
\hline NEKnRTC & 94.80 & 92.62 & 84.97 & 92.28 & 7 & 99.89 & 81.38 & 83.19 & 88.91 & 7 \\
\hline NBSTC & 70.14 & 71.70 & 57.45 & 66.52 & 15 & 55.96 & 65.70 & 70.54 & 66.60 & 15 \\
\hline BSRTC & 90.07 & 100.00 & 100.00 & 88.11 & 12 & 84.87 & 100.00 & 100.00 & 87.18 & 10 \\
\hline
\end{tabular}

Table 3. Overall performance scores of the sample STUs for select years based on combined output.

\begin{tabular}{|c|c|c|c|c|c|c|c|c|c|c|c|c|c|c|c|}
\hline & APSRTC & MSRTC & KnSRTC & NWKnRTC & GSRTC & UPSRTC & RSRTC & STHAR & SBSTC & KDTC & OSRTC & KSRTC & NEKnRTC & NBSTC & BSRTC \\
\hline 2003-04 & 100.0 & 91.5 & 100.0 & 100.0 & 92.9 & 98.9 & 100.0 & 93.9 & 81.4 & 100.0 & 100.0 & 100.0 & 100.0 & 70.2 & 90.1 \\
\hline 2008-09 & 100.0 & 87.8 & 97.1 & 87.5 & 100.0 & 100.0 & 100.0 & 100.0 & 78.7 & 98.1 & 100.0 & 76.5 & 92.6 & 71.7 & 100.0 \\
\hline 2013-14 & 100.0 & 94.4 & 100.0 & 97.5 & 100.0 & 100.0 & 100.0 & 86.5 & 100.0 & 63.6 & 100.0 & 86.1 & 85.4 & 70.5 & 100.0 \\
\hline Mean (2003-14) & 100.0 & 89.7 & 99.1 & 92.4 & 96.5 & 99.0 & 100.0 & 94.1 & 82.7 & 91.2 & 100.0 & 87.3 & 92.9 & 69.2 & 88.4 \\
\hline Performance Rank & 1 & 11 & 4 & 9 & 6 & 5 & 1 & 7 & 14 & 10 & 1 & 13 & 8 & 15 & 12 \\
\hline
\end{tabular}

only five STUs. In general, STUs' effectiveness score is lower than their efficiency score. Table 2 shows that the average effectiveness score exceeds the average efficiency score only for two out of fifteen sample STUs, KnSRTC and KSRTC. Three STUs have almost identical scores for both efficiency and effectiveness whereas remaining ten STUs have lower effectiveness score.

STUs are also evaluated based on overall relative performance scores, obtained in dual output scenario, across the temporal and cross sectional dimensions. The overall performance scores of the sample STUs are calculated for all the years from 2003-04 to 2013-14 and reported for selected years in Table 3 along with mean performance scores, calculated as the arithmetic average of the annual performance scores and STUs are ranked accordingly. Table 3 reveals that the three STUs, APSRTC, RSRTC, and OSRTC, achieved perfect score of $100 \%$ during every year of the sample period. NBSTC (69.2\%), SBSTC (82.7\%), 
KSRTC (87.3\%), BSRTC (88.4\%), and MSRTC (89.7\%) are among the worst performers having performance score of less than $90 \%$. This shows that the state owned bus transport operators operating in the state of West Bengal, Bihar, Kerala, and Maharashtra need to improve their performance significantly. They can learn from the operators operating in the state of Andhra Pradesh, Rajasthan, and Orissa.

An important research question in transportation research, dealt in this paper, is whether there is a correlation between effectiveness and efficiency. Effectiveness and efficiency are, in fact, positively correlated with a correlation coefficient of 0.768. Effectiveness and overall performance score are positively correlated with a correlation coefficient of 0.893 . Efficiency, on the other hand, is highly correlated with overall performance score with a correlation coefficient of 0.928 . The results are summarized in correlation matrix presented in Table 4.

One of the most important research focus of this paper, not so well explored in the transportation sector research in India, is the establishment of a connect between quantitative measure of scale elasticity and firm size. The results of scale elasticity or returns to scale estimation are discussed next followed by a discussion on firm size and scale elasticity relationship. A value of one denotes constant returns to scale, a value greater than one indicates increasing returns to scale and a value less than one indicates decreasing returns to scale.

Table 5 reports the quantitative measures of scale elasticities for sample STUs for selected years. Figures show that seven out of fifteen STUs operate on increasing returns to scale, same number of STUs operate on decreasing returns to scale, and one STU, RSRTC, operates on constant returns to scale. As expected, we found a negative relationship between size of the STUs, measured in terms of passenger-km served, and the scale elasticity. The correlation coefficient between these two comes out to be -0.583 . The relationship is moderate and is statistically significant with a t-statistic of 2.585 .

We have also segregated the STUs, using k-means clustering in three sizes: Large, Medium and Small. A summary statistics for the scale elasticity estimates, with STUs being grouped based on their size, is given in Table 6. It shows that the medium size STUs, such as RSRTC, are operating at close to optimal scale. Large size firms, such as APSRTC and MSRTC, are showing decreasing returns whereas small size firms, such as BSRTC, NBSTC, SBSTC, and KDTC, are showing increasing returns to scale. This means that both large as well as small size STUs are operating at non-optimal scale. Since RSRTC is operating at the most productive scale size, optimal fleet size for STUs would be the fleet size of

Table 4. Correlation statistics for performance measure.

\begin{tabular}{cccc}
\hline & Efficiency & Effectiveness & Combined \\
\hline Efficiency & 1 & 0.768 & 0.928 \\
Effectiveness & & 1 & 0.893 \\
Combined & & & 1 \\
\hline
\end{tabular}


Table 5. Scale elasticity measures for selected years and mean for 2003-14 for sample STUs.

\begin{tabular}{ccccc}
\hline STUs & $2003-04$ & $2008-09$ & $2013-14$ & Mean \\
\hline APSRTC & 0.903 & 0.958 & 0.953 & 0.947 \\
MSRTC & 0.974 & 0.993 & 0.926 & 0.978 \\
KnSRTC & 0.961 & 0.866 & 0.809 & 0.916 \\
NWKnRTC & 1.000 & 1.013 & 1.007 & 0.996 \\
GSRTC & 0.997 & 1.000 & 1.000 & 0.994 \\
UPSRTC & 1.011 & 0.797 & 1.000 & 0.882 \\
RSRTC & 1.000 & 1.000 & 1.000 & 1.000 \\
STHAR & 1.020 & 1.000 & 1.041 & 1.024 \\
SBSTC & 1.213 & 1.299 & 1.085 & 1.246 \\
KDTC & 1.303 & 1.480 & 1.310 & 1.495 \\
OSRTC & 1.271 & 1.000 & 0.863 & 1.088 \\
KSRTC & 1.000 & 1.020 & 0.995 & 1.013 \\
NEKnRTC & 1.000 & 1.019 & 1.024 & 0.986 \\
NBSTC & 1.151 & 1.226 & 1.070 & 1.245 \\
BSRTC & 1.556 & 1.633 & 1.867 & 1.517 \\
Overall Mean & 1.091 & 1.087 & 1.063 & 1.088 \\
\hline
\end{tabular}

Table 6. Summary statistics of scale elasticity, STUs grouped on size.

\begin{tabular}{cccc}
\hline & Large & Medium & Small \\
\hline Mean & 0.952 & 0.968 & 1.223 \\
Median & 0.958 & 1.000 & 1.213 \\
Coefficient of Variation & 0.041 & 0.093 & 0.189 \\
\hline
\end{tabular}

the RSRTC, that is around 4500 to 5000 buses. Since fleet strength of large size STUs, such as APSRTC $(22,145)$ and MSRTC $(18,055)$, is far more than the optimal one, the division of these STUs would lead to higher level of productivity. On the other hand, smaller STUs operating in the same state, such as NBSTC and SBSTC of West Bengal, may be merged for the same reason.

\section{Conclusions}

DEA, as a technique of benchmarking and relative performance evaluation of state owned utilities, like STUs, is a frequently used tool in applied economic research. In this paper, we have used an output oriented DEA methodology with VRS assumption to estimate the efficiency, effectiveness, and overall performance scores of fifteen major STUs in India. VRS is, in fact, an appropriate assumption because our analysis revealed that most of the STUs are operating at scale elasticities different from unity. We found that the three STUs, APSRTC, RSRTC, and OSRTC, are the most efficient ones with $100 \%$ efficiency score 
whereas NBSTC (66.52\%), KSRTC (71.70\%), and SBSTC (78.94\%) are among the least efficient ones. Moreover, APSRTC and OSRTC not only achieved $100 \%$ efficiency score but also $100 \%$ effectiveness score whereas NBSTC (66.60\%) and SBSTC (74.20\%) along with MSRTC (72.82\%) are among the least effective firms. This shows that the STUs operating in West Bengal, NBSTC and SBSTC, are not only the least efficient but also the least effective whereas Andhra Pradesh state road transport corporation, which is the largest bus transport operator in the world, is the most efficient and effective operator. That's why, in general, there is a strong positive correlation between STUs' efficiency and their effectiveness. We also evaluated STUs based on overall relative performance obtained in dual output scenario, bus-km as well as passenger-km. We found that the three STUs, APSRTC, RSRTC, and OSRTC, achieved perfect score of $100 \%$ whereas both the STUs of West Bengal, NBSTC (69.2\%) and SBSTC (82.7\%) performed worse than others.

The second part of the research concentrated on estimating the returns to scale and its relationship with firm size. The main purpose is to look for those STUs which are operating at or close to optimal size. We found that there is negative relationship between size of the STUs and returns to scale; large size firms are showing decreasing returns to scale whereas small size ones are operating on increasing returns to scale. In general, medium size firms such as RSRTC, NWKnRTC, NEKnRTC, and STHAR are operating with constant returns to scale. This means that both large as well as small size STUs are operating at non-optimal scale. We found that the optimal fleet size for STUs would be around 4500 to 5000 buses. Since fleet strength of some of the large size firms is far more than the optimal one, their demerger would be desirable and likely to lead to higher level of productivity. On the other hand, smaller STUs operating in the same state, such as NBSTC and SBSTC of West Bengal, should be merged. This will be in the larger interest of the public, as STUs are in general, continuously making substantial losses. A size correction through mergers, demergers or altering scale of operation, as the case may be, will be economically prudent.

\section{Acknowledgements}

This paper is part of a seed money project sponsored by the Indian Institute of Management, Lucknow, India. We are thankful to the Director and Dean (Research) of the institute for providing grant for the study.

\section{References}

[1] Ferrier, G.D. and Lovell, C.A.K. (1990) Measuring Cost Efficiency in Banking: Econometric and Linear Programming Evidence. Journal of Econometrics, 46, 229 245. https://doi.org/10.1016/0304-4076(90)90057-Z

[2] Bjurek, H., Hjalmarsson, L. and Forsund, F.R. (1990) Deterministic Parametric and Nonparametric Estimation of Efficiency in Service Production: A Comparison. Journal of Econometrics, 46, 213-227. https://doi.org/10.1016/0304-4076(90)90056-Y 
[3] Lovell, C.K. (1993) Production Frontiers and Productive Efficiency. In: Fried, H.O., Lovell, C.K. and Schmidt, S.S., Eds., The Measurement of Productive EfficiencyTechniques and Applications, Oxford University Press, Oxford, 3-35.

[4] Cullinane, K., Wang, T.F., Song, D.W. and Ji, P. (2006) The Technical Efficiency of Container Ports: Comparing Data Envelopment Analysis and Stochastic Frontier Analysis. Transportation Research Part A, 40, 354-374.

https://doi.org/10.1016/j.tra.2005.07.003

[5] Jarboui, S., Forget, P. and Boujelbene, Y. (2012) Public Road Transport Efficiency: A Literature Review via the Classification Scheme. Public Transport, 4, 101-128. https://doi.org/10.1007/s12469-012-0055-3

[6] Aigner, D., Lovell, C.A.K. and Schmidt, P. (1977) Formulation and Estimation of Stochastic Frontier Production Function Models. Journal of Econometrics, 6, 21-37. https://doi.org/10.1016/0304-4076(77)90052-5

[7] Battese, G.E. and Coelli, T.J. (1992) Frontier Production Functions, Technical Efficiency and Panel Data: With Application to Paddy Farmers in India. In: Gulledge, T.R. and Lovell, C.A.K., Eds., International Applications of Productivity and Efficiency Analysis, Springer, Dordrecht, 149-165. https://doi.org/10.1007/978-94-017-1923-0_10

[8] Battese, G.E. and Coelli, T.J. (1995) A Model for Technical Inefficiency Effects in a Stochastic Frontier Production Function for Panel Data. Empirical Economics, 20, 325-332. https://doi.org/10.1007/BF01205442

[9] Farrell (1957) The Measurement of Productive Efficiency. Journal of the Royal Statistical Society, Series A (General), 120, 253-290. https://doi.org/10.2307/2343100

[10] Charnes, A., Cooper, W.W. and Rhodes, E. (1978) Measuring the Efficiency of Decision Making Units. European Journal of Operational Research, 2, 429-444. https://doi.org/10.1016/0377-2217(78)90138-8

[11] Banker, R.D., Charnes, A. and Cooper, W.W. (1984) Some Models for Estimating Technical and Scale Inefficiencies in Data Envelopment Analysis. Management Science, 30, 1078-1092. https://doi.org/10.1287/mnsc.30.9.1078

[12] Lovell, C.A.K. and Rouse, A.P.B. (2003) Equivalent Standard DEA Models to Provide Super-Efficiency Scores. Journal of the Operational Research Society, 54, 101-108. https://doi.org/10.1057/palgrave.jors.2601483

[13] Kumbhakar, S.C. and Bhattacharyya, A. (1996) Productivity Growth in Passenger-Bus Transportation: A Heteroskedastic Error Component Model with Unbalanced Panel Data. Empirical Economics, 21, 557-573. https://doi.org/10.1007/BF01180701

[14] Jørgensen, F., Pedersen, P.A. and Volden, R. (1997) Estimating the Inefficiency in the Norwegian Bus Industry from Stochastic Cost Frontier Models. Transportation, 24, 421-433. https://doi.org/10.1023/A:1004975000392

[15] Viton, P.A. (1997) Technical Efficiency in Multi-Mode Bus Transit: A Production Frontier Analysis. Transportation Research Part B: Methodological, 31, 23-39.

[16] Jha, R. and Singh, S.K. (2001) Small Is Efficient: A Frontier Approach to Cost Inefficiencies in Indian State Road Transport Undertakings. International Journal of Transport Economics/ Rivista internazionale di economia dei trasporti, 28, 95-114.

[17] Singh, S.K. and Venkatesh, A. (2003) Comparing Efficiency across State Transport Undertakings: A Production Frontier Approach. Indian Journal of Transport Management, 27, 374-391

[18] Karlaftis, M.G. (2003) Investigating Transit Production and Performance: A Pro- 
gramming Approach. Transportation Research Part A: Policy and Practice, 37, 225-240.

[19] Boame, A.K. (2004) The Technical Efficiency of Canadian Urban Transit Systems. Transportation Research Part E: Logistics and Transportation Review, 40, 401-416.

[20] Karlaftis, M.G. (2004) A DEA Approach for Evaluating the Efficiency and Effectiveness of Urban Transit Systems. European Journal of Operational Research, 152, 354-364.

[21] Charnes, A., Gallegos, A. and Li, H. (1996) Robustly Efficient Parametric Frontiers via Multiplicative DEA for Domestic and International Operations of the Latin American Airline Industry. European Journal of Operational Research, 88, 525-536.

[22] Odeck, J. and Alkadi, A. (2004) The Performance of Subsidized Urban and Rural Public Bus Operators: Empirical Evidence from Norway. The Annals of Regional Science, 38, 413-431. https://doi.org/10.1007/s00168-003-0152-y

[23] Sampaio, B.R., Neto, O.L. and Sampaio, Y. (2008) Efficiency Analysis of Public Transport Systems: Lessons for Institutional Planning. Transportation Research Part A: Policy and Practice, 42, 445-454.

[24] Saxena, P. and Saxena, R.R. (2010) Measuring Efficiencies in Indian Public Road Transit: A Data Envelopment Analysis Approach. Opsearch, 47, 195-204. https://doi.org/10.1007/s12597-011-0034-5

[25] Agarwal, S., Yadav, S.P. and Singh, S.P. (2010) DEA Based Estimation of the Technical Efficiency of State Transport Undertakings in India. Opsearch, 47, 216-230. https://doi.org/10.1007/s12597-011-0035-4

[26] Jordá, P., Cascajo, R. and Monzón, A. (2012) Analysis of the Technical Efficiency of Urban Bus Services in Spain Based on SBM Models. ISRN Civil Engineering, 2012, 1-13. https://doi.org/10.5402/2012/984758

[27] Vaidya, O.S. (2014) Evaluating the Performance of Public Urban Transportation Systems in India. Journal of Public Transportation, 17, 174-191. https://doi.org/10.5038/2375-0901.17.4.10

[28] Hanumappa, D., Ramachandran, P., Sitharam, T.G. and Lakshmana, S. (2015) Performance Evaluation of Bangalore Metropolitan Transport Corporation: An Application of Data Envelopment Analysis. Journal of Public Transportation, 18, 1-19. http://www.scopus.com/inward/record.url?eid=2-s2.0-84931461965\&partnerID=40 \&md5=18348f6e4bce26d0a9dce6c90835f5b8 https://doi.org/10.5038/2375-0901.18.2.1

[29] Venkatesh, A. and Kushwaha, S. (2017) Measuring Technical Efficiency of Passenger Bus Companies in India: A Non-Radial Data Envelopment Analysis Approach. Opsearch, 1-18. https://doi.org/10.1007/s12597-017-0303-Z

[30] Sahoo, B.K. and Tone, K. (2015) Scale Elasticity in Non-Parametric DEA Approach. In: Zhu, J., Ed., Data Envelopment Analysis, Springer, Boston, 269-290. https://doi.org/10.1007/978-1-4899-7553-9_9

[31] Panzar, J.C. and Willig, R.D. (1977) Economies of Scale in Multi-Output Production. The Quarterly Journal of Economics, 91, 481-493. https://doi.org/10.2307/1885979 